\title{
P-PI and super twisting sliding mode control schemes comparison for high-precision CNC machining
}

\author{
Luis Rubio \\ Research Associate \\ Centre for precision manufacturing \\ University of Strathclyde \\ Glasgow, UK, G1 1XJ \\ Luis.rubio@strath.ac.uk
}

\author{
Asier Ibeas \\ Associate Professor \\ Department of Telecommunications \\ and Systems Engineering \\ Autonomous University of \\ Barcelona \\ Barcelona, Spain, 08193
}

\author{
Xichun Luo \\ Professor \\ Centre for precision manufacturing \\ University of Strathclyde \\ Glasgow, UK, G1 1XJ \\ Xichun.luo@strath.ac.uk
}

\begin{abstract}
Multi-axis high precision machining uses linear motors actuators in order to deal with robustness and stability in the broad range of cutting conditions. Currently, Computer Numerical Controls (CNCs) integrate PID type controllers in order to deal with tracking errors and disturbances. Moreover, CNCs introduce feed-forward control loop to cope with model variations. However, to overcome the influences of disturbances and model uncertainties natural control approach is adopted by sliding mode controller (SMC). This paper proposes a supertwisting sliding mode control algorithm to cope with the switching control for keeping the dynamics of the system within the designed requirements. Furthermore, the paper compares the behaviour of P-PI position-velocity control approach and supertwisting SMC. The implementation and evaluation of the algorithms in Matlab shows that super-twisting SMC is able to track the reference signal more accurate and robustness against the estimated processing parameters and disturbances. The main source of instability in sliding mode controller knowing as chattering is minimized when applied the super-twisting control algorithm.
\end{abstract}

Keywords; Tracking control, Micro-milling, Manufacturing Automation, PID control, Sliding Mode Control, Computer Numerically Control (CNC)

\section{INTRODUCTION}

The tendency to miniaturization is leading to an increasing demand on high precision machining. To achieve high precision machining, the motion of each axis needs to be controlled smoothly and precisely, so they will follow the preprogrammed desired trajectory accurately. When using linear motors for machining, three variables have to be considered, namely, mass, damping and disturbance acting on the system. Typically, machine mass or damping do not change unexpectedly during machining. The change of plant parameters results from the variations of mass of workpiece and use of lubricants. Furthermore, cutting forces are the main source of disturbances in machining when linear motor actuators are used. As a consequence, cutting forces are required to be rejected, particularly in the lower frequency ranges where the system stiffness is a subject for control [1]. These are the most likely source of unpredicted plant variations, and the most likely cause of unexpected plant behaviour.

Nowadays commercial CNC machines are composed by feedback controller which deals with the traditional transitory behaviour and steady state errors. Proportional, integral and derivative (PID) control algorithm is commonly used on the feedback controllers. The poor tracking performance at corners and on non-linear paths [1] offered by feedback controllers made to introduce feed forward control algorithms. The performance of feed forward controllers can be degraded due to a model mismatch of the system (i.e., the model does not represent accurately the actual system) and the mathematical complexity induced by the calculation of the inverse of the transfer function. As a result, in practical situation the performance of this pre-compensation approach is not so very satisfactory.

To overcome the influences of disturbances and model uncertainties, known as variable structure systems, natural control approach is adopted by sliding mode controller (SMC) which was first proposed by Utkin [2]. The controller gives some desirable closed-loop properties, including invariance, order reduction and robustness against parameters variations and disturbances. The sliding mode control exhibits low sensibility with respect to different types of uncertainties in operation conditions such as parameter variations and unknown disturbances [2]. The design of SMC consists of two stages, i.e. an equivalent control and a switching control. The equivalent control is derived from the definition of sliding surface, to which the controlled system trajectories must belong, and it is designed in accordance with certain performance criterion. The second stage consists of switching control which forces the system to slide along the sliding surface. When sliding mode is realized, the system exhibits robustness properties with respect to parameter perturbations and external matched disturbances. As switching control usually takes a discontinuous form, it often results in undesirable chattering in the control signals [3].

In this paper, typical PID-based control scheme used in literature knowing as P-PI position-velocity control scheme and the third generation of sliding mode controller, called 
super-twisting sliding mode controls are analysed and compared proposed when controlling linear motors in multiaxis machine tools.

The aim of the paper is to show the advantages of using more sophisticated control algorithms in high precision machining in order to achieve better performances indexes in terms of robustness and quality of tracking the reference signal and accuracy of the final products.

The paper is divided into several parts. Section 2 models linear motors for their application in machining and perturbations incurred by cutting forces. Section 3 presents the P-PI position-velocity control scheme. Section 4 introduces supertwisting SMC algorithm justifying its stability. Section 5 introduces the selection of the sliding mode surface. Section 6 presents simulation results and section 7 concludes the paper.

\section{SYSTEM MODELLING}

The current section introduces the modelling of linear motors driven by direct drives. As each motor work independently, it is intended to track-control at each motor. Moreover, the cutting forces which act as a perturbation are represented and explained in the second sub-section.

\section{A. Linear motor model}

The model of the linear motor direct drive feed system is established according to its work principle [4,5]. The equations which govern the movement of the linear motor are considered to be modelled by the second order mass-damping equation:

$$
m \ddot{x}+b \dot{x}=F
$$

Where $x=x(t)$ denotes the position and $F$ is the external control force, $m(K g)$ is the actual moving mass (table and workpiece), and $b(\mathrm{Kg} / \mathrm{m})$ is the actual damping coefficient of the guide-ways.

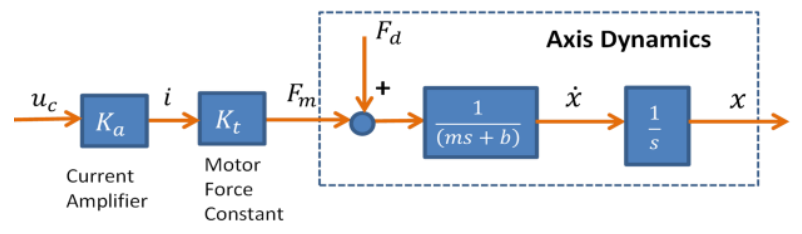

Figure 1: Block diagram of a feed drive machine tool

The block diagram is shown in figure 1 in which $1 /(m s+b)$ represents the Laplace transformation of equation (1). The movement of a single axis of the machine tool driven by linear motor is then represented by current amplifier, motor force constant, and axis dynamics including disturbance forces. In the block diagram $u_{c}(t), i(t), F_{m}(t)$ and $F_{d}(t)$ represents the control signal, the driven current, the force given by the linear motor and the disturbance force, respectively.

\section{B. Disturbances model}

The main source of disturbance in machine tools driven by linear motors is given by the cutting forces. Previous studies have addressed the calculation of cutting forces, in which mechanistic approaches are mainly used [6]. In this section the model proposed in [7] is used to represent the cutting forces in micro-milling process. The model integrates the methodology from conventional milling mechanism with the true undeformed chip thickness and tool run out effect. By this way, this model is able to link the cutting forces with geometrical aspects of micro milling operation and mechanical behaviour of materials in micro milling. For simplicity, 2D orthogonal cutting is assumed. By neglecting the axial forces, the cutting forces are expressed as:

$$
\begin{aligned}
F_{t} & =K_{t}(h) h w \\
F_{n} & =K_{n}(h) h w
\end{aligned}
$$

Where $F_{t}$ and $F_{n}, h$ and $w$ are the tangential and normal force component, the uncut chip thickness and the width of cut, respectively. Since the uncut chip thickness and instantaneous cutting force coefficients are determined, the tangential $\left(F_{t}\right)$ and normal (or radial, $F_{n}$ ) forces acting on the cutting edge with different axial depth of cut $(a)$ are expressed as

$\left.\begin{array}{l}d F_{t}=K_{t} h(\phi) d a \\ d F_{n}=K_{n} h(\phi) d a\end{array}\right\}$

Where $\phi$ is the instantaneous angular position of the tool edge, $K_{t}$ and $K_{n}$ are the instantaneous cutting force coefficients and the integration of the helix angle can be considered between start and exit angular angles.

Finally, tangential and normal force components are projected into the $\mathrm{x}$ and $\mathrm{y}$ axis as:

$$
\left.\begin{array}{c}
F_{x}=-F_{t} \cos \phi-F_{n} \sin \phi \\
F_{y}=F_{t} \sin \phi-F_{n} \cos \phi
\end{array}\right\}
$$

\section{P-PI POSITION VELOCITY CONTROL SCHEME}

One of the most typical control schemes to deal with positioning using PID controllers is the P-PI control scheme presented in this section. This scheme allows practitioners to manage controlling position and velocity of the actuators programming gains at the same time. This scheme (figure 2) has been considered in previous research outputs in literature, for example in [4].

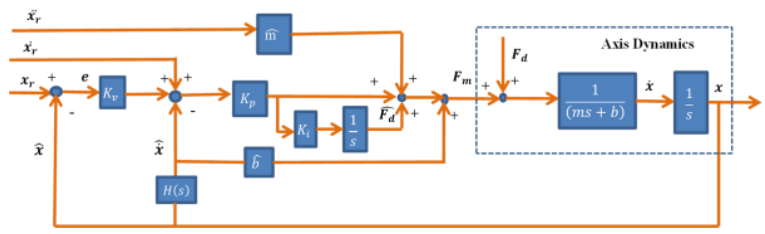

Figure 2: P-PI position-velocity control scheme A proportional gain feedback the position and velocity error and proportional gain in the velocity is integer in order to estimate and compensate perturbations. A feed-forward velocity and acceleration controller is introduced to deal with variations in the mass and damping of the system.

The equations of movement in the control loop diagram are described as:

$m \ddot{x}+b \dot{x}=F(t)$

$F(t)=F_{d}(t)+F_{m}(t)$

$F_{m}(t)=F_{a}(t)+F_{b}(t)$ 
$F_{a}(t)=\widehat{m} x_{\text {ref }}^{\ddot{*}}+K_{p} \beta+\widehat{F_{d}}$

$F_{b}(t)=\hat{b} \hat{\dot{x}}$

$\beta=K_{v}\left(x_{\text {ref }}-\hat{x}\right)+\left(x_{\text {ref }}^{\cdot}-\hat{\dot{x}}\right)$

$\widehat{F_{d}}=\int K_{p} K_{i} \beta d x$

Where $x_{\text {ref }}, x_{\text {ref }}^{\cdot}$ and $x_{\text {ref }}^{. *}$ are the position, velocity and acceleration references, $\hat{m}$ the estimated mass, $\hat{b}$ the estimated damping, $K_{v}$ position proportional gain, $K_{p}$ velocity proportional gain and $K_{i}$ velocity integral gain.

\section{SUPER TWISTING SMC ALGORITHM}

The control signal of the SMC is normally split out into two parts, one regarding the equivalent control, which deals with the dynamics of the system and the sliding surface, and another regarding the switching control which is responsible for keeping the dynamics of the system onto the sliding surface. So,

$F=F^{e q}+F^{s w}$

The equivalent control $F^{e q}$ relies on the dynamics of the system and the sliding surface, $S(t)$. The approach taken into consideration in this section is totally classical and wellknown in the literature $[8,9,10]$. Consider the PID-type sliding surface:

$S(t)=\dot{e}(t)+\mu e(t)+\lambda \int_{0}^{t} e(\tau) d \tau$

With $e(t)=x_{r e f}(t)-x(t)$, and $\mu$ and $\lambda$ are positive design parameters. The control gains $\mu$ and $\lambda$ should be chosen so the characteristic polynomial $s^{2}+\mu s+\lambda=0 \quad(s$ denotes a complex variable here) is strictly Hurwitz, i.e., a polynomial with roots located strictly in the open left half of the complex plane. Its time derivative is given by:

$\dot{S}(t)=\ddot{e}(t)+\mu \dot{e}(t)+\lambda e(t)$

The control objective is imposed by $\dot{S}(t)=0$, which will give the solution of the equivalent control. Thus,

$\dot{S}(t)=0=\ddot{x}_{r e f}(t)-\ddot{x}(t)+\mu\left(\dot{x}_{r e f}(t)-\dot{x}(t)\right)+$

$\lambda\left(x_{\text {ref }}(t)-x(t)\right)$

Introducing (1) in (8), it results in:

$\ddot{x}_{\text {ref }}(t)-\frac{1}{m}(F-b \dot{x}(t))+\mu\left(\dot{x}_{\text {ref }}(t)-\dot{x}(t)\right)+$

$\lambda\left(x_{\text {ref }}(t)-x(t)\right)=0$

While isolated, selecting $\mu=\frac{b}{m}$ (bandwidth of the system, $\mathrm{rad} / \mathrm{sg}$ ), the control signal can be expressed as:

$F=m\left(\ddot{x}_{r e f}(t)+\frac{b}{m} \dot{x}_{r e f}(t)+\lambda e(t)\right)$

Since the actual parameters of the system are not known, their estimates have to be used while, in addition, a switching term is added to cope with uncertainties and to drive the system into the sliding surface. Therefore, the complete sliding control law reads:

$F=\widehat{m}\left(\ddot{x}_{\text {ref }}(t)+\frac{\hat{b}}{\hat{m}} \dot{x}_{\text {ref }}(t)+\lambda e(t)\right)+\Lambda \sqrt{|S(t)|} \operatorname{sign}(S)+$ $v(t), \Lambda>0$

With $\dot{v}(t)=\operatorname{sign}(S)$. This controller equation guarantees the continuity of the control law and allows avoiding/reducing the chattering. Chattering is quite undesirable as it will accelerate the wear and tear of actuators. With the same Lyapunov candidate function as before it is obtained:

$$
\begin{aligned}
& \dot{V}(t)=S\left[\left(1-\frac{\widehat{m}}{m}\right) \ddot{x}_{r e f}(t)+\left(\frac{\hat{b}}{\widehat{m}}-\frac{b}{m}\right) \dot{e}(t)+\right. \\
& \left.\lambda\left(1-\frac{\widehat{m}}{m}\right) e(t)\right]-\frac{\Lambda}{m} \sqrt{|S(t)||S(t)|-} \\
& S \int_{0}^{t} \operatorname{sign}(S(\tau)) d \tau
\end{aligned}
$$

When the system parameters are known, the above equation can be simplified as, $\dot{V}(t)=-\frac{\Lambda}{m} \sqrt{|S(t)| \mid}|S(t)|-$ $S \int_{0}^{t} \operatorname{sign}(S(\tau)) d \tau$, which is negative definite, and implies the stability of the closed-loop according to the second Lyapunov theorem. When the system is unknown, $\Lambda$ must be larger enough to make the term $\frac{\Lambda}{m}$ larger than the uncertainty in the first term so that the Lyapunov function is a negative definite. Thus, for sufficiently large values of $\Lambda$ the closed loop system is stable.

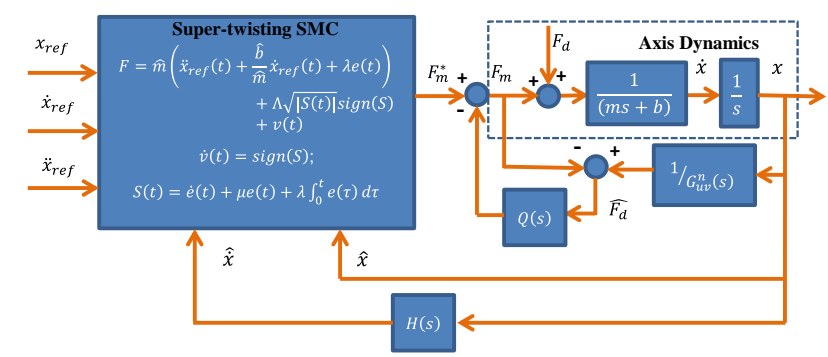

Figure 3: Super-twisting SMC control scheme with disturbance estimation

Figure 3 represents the integration of the super-twisting control algorithm and disturbance estimation including the system dynamics and cutting force perturbation. The detailed explanation of the disturbance observer has not been included in this paper but the interested reader can consult reference [10] which the steps on how to design the observer. The reasons for including an observer are to understand and avoid the influence of disturbance in the system. The disturbance observer can be designed in many ways [10]:

- It may be considered as a lead/lag compensator by appropriately designing the parameters of the filter.

- Adjusting the filter of the disturbance observer to satisfy requirements for robustness and disturbance suppression characteristics. The system sensitivity function and the system transmission function can thus be set independently. Such a feature is especially useful when there are strict requirements on both set point tracking and disturbance suppression and an acceptable compromise between these sometimes conflicting requirements might not exist.

- Carry out simulation and fine tuning until the performance is acceptable.

References from required trajectories in position, velocity and acceleration are provided from the CAD/CAM systems or programmed by the engineer. These trajectories may be designed in order to fulfil specifications to get smoothness, such as, jerk or snap limited trajectories [11]. In order to test the proposed control algorithm sufficient excited signals in the 
form of sinusoidal waves are chosen as position reference signals, considering their first and second derivate as velocity and acceleration reference signals.

The control algorithm takes the reference signals with certain initial conditions for the position, velocity and acceleration and processes the super-twist SMC control algorithm in a certain sliding surface to generate the control signal, $F_{m}^{*}$. The estimation of the forces given by the disturbance observed is processed in parallel and it is subtracted to the super-twist algorithm. Finally, the output position is measured and velocity is estimated. In this case, for estimating the velocity from the output position a first order filter is used. And the super-twisting SMC is processed again.

\section{RESULTS}

The simulations were implemented in Matlab/Simulink environment using the integrated numerical method for both P-PI and SMC control approaches. Continuous time domain simulations are typical approaches in control systems theory in order to analyse the behaviour of the system.

Extensive simulations have been carried out in order to evaluate the proposed control algorithm. The following parameters and assumptions have been taken into consideration in the simulation, actual mass and damping $m=10 \mathrm{Kg}, B=250 \mathrm{Kg} / \mathrm{s}$, and amplifier and motor force constant $k_{t}=2 A / V, K_{a}=50 \mathrm{~N} / A$. The parameters of the plant change slight with time due to variations in the mass as cutting process, variations in the damping due to lubricant and uncertainties in their values. In the simulations the estimated mass is considered as $\widehat{m}=11 \mathrm{Kg}$ and estimated damping $\hat{B}=255^{\mathrm{Kg}} / \mathrm{s}$. Moreover, a sufficient excited input signal by means of a sinusoidal input of $1 \mu \mathrm{m}$ of amplitude and $3 \mathrm{rad} / \mathrm{s}$ of frequency is taking into account. The cutting conditions of the micro-milling process are spindle speed, $\Omega=11520 \mathrm{rpm}$, axial depth of cut, $w=0.05 \mathrm{~mm}$, cutter diameter, $d=0.09 \mathrm{~mm}$ and number of teeth, $N_{t}=2$.
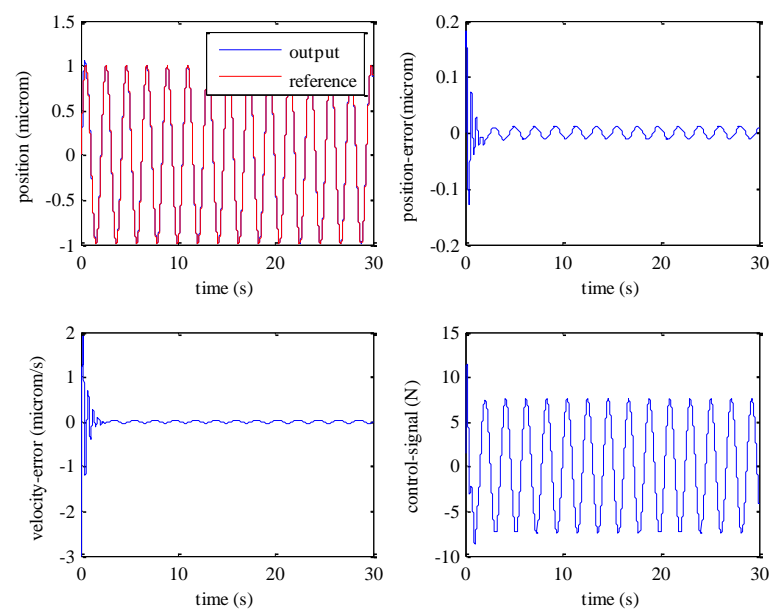

Figure 4: P-PI control scheme without disturbance
Figures 4 and 5 outputs the results of the P-PI control scheme when external disturbances are not influencing the system and when they are influencing respectively. It can be observed fluctuations in the transitory responses of the position errors and velocity errors. Moreover, the velocity error is not a smooth signal which can give problems in the realization of the system loop. Finally, the system is not stable for the broad range of parameters $k_{v}, k_{p}$ and $k_{i}$, under certain programmed values of these gains the system can lead to be unstable. $k_{v}=30, k_{p}=50$ and $k_{i}=0.5$ have been used for simulation purposes.
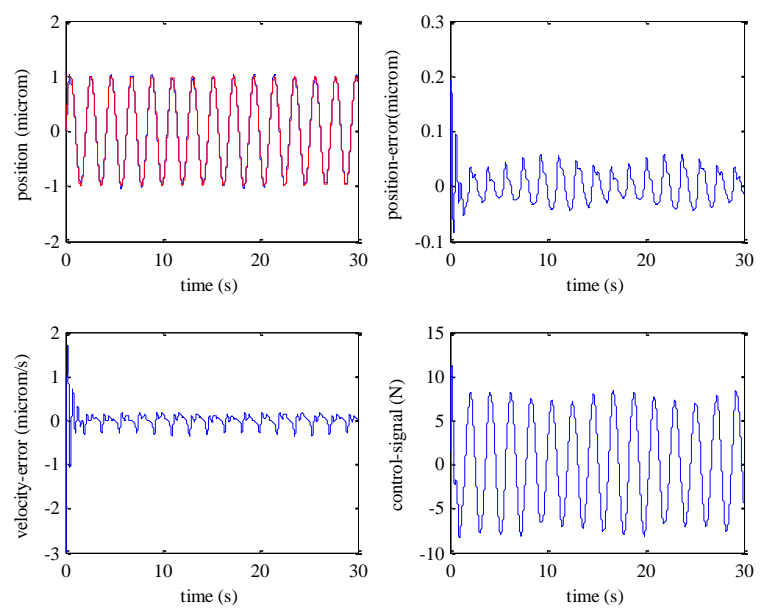

Figure 5: P-PI control scheme with the influence of external disturbances

Figures 6, 7 and 8 shows the super-twisting SMC control scheme for the cases when disturbances are not acting in the systems, disturbances are acting into the system but not disturbance estimator is in the control loop and disturbances are acting and disturbance estimator is included in the control loop, respectively.
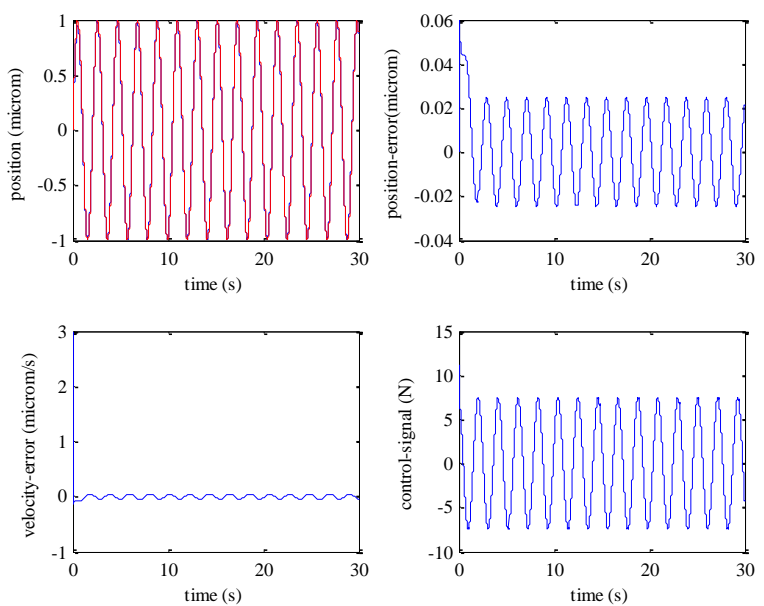

Figure 6: Super twisting SMC without disturbance

When using the super twisting SMC control scheme the transitory response of the position error and velocity error are smoother than in the previous case. Moreover, the influence of 
the disturbances is less than when using the P-PI control scheme. As a result, smoother tool paths are achieving when using SMC schemes respect to PID-based schemes. Finally, figure 8 demonstrates that the use of an estimator can be damp the effect of the perturbation acting on the system.
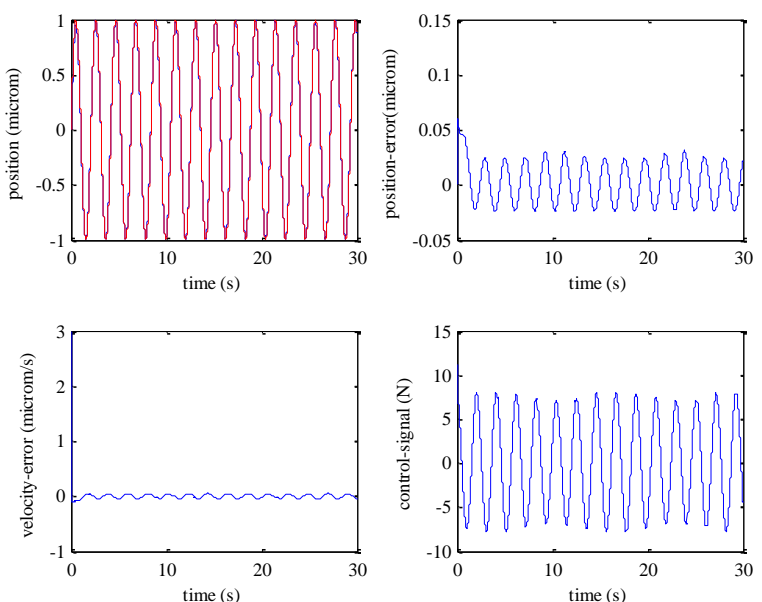

Figure 7: Super twisting SMC with the influence of external disturbances but not disturbance estimator included in the control loop
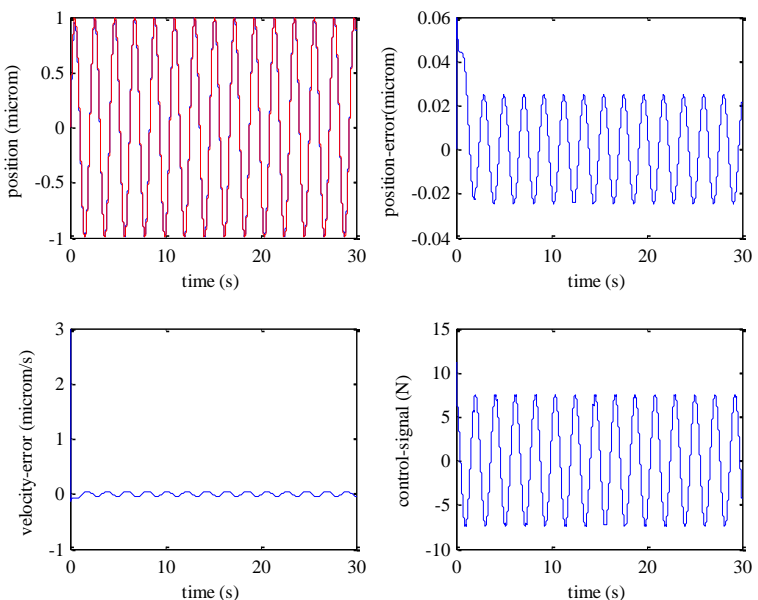

Figure 8: Super twisting SMC with the influence of external disturbances and disturbance estimator included in the control loop

Table I: Comparison between both control schemes

\begin{tabular}{|c|c|c|c|c|}
\multicolumn{1}{c}{ Position error } & \multicolumn{1}{c|}{ Velocity error } \\
\hline & $\max$ & $\sigma$ & $\max$ & $\sigma$ \\
\hline P-PI without disturbances (fig.4) & 0.1826 & 0.0178 & 1.9014 & 0.2096 \\
\hline P-PI with disturbances (fig.5) & 0.1895 & 0.0307 & 1.6910 & 0.2339 \\
\hline SMC without disturbances (fig. 6) & 0.059 & 0.0186 & 3 & 0.0790 \\
\hline SMC with disturbances (fig. 7) & 0.0604 & 0.0189 & 3 & 0.0855 \\
\hline
\end{tabular}

Table I gives the values of the maximum and standard deviation $(\sigma)$ of the position and velocity errors. Position errors have less overshoots when super twisting SMC is applied but bigger velocity errors in spite of having less oscillatory transitory responses. The standard deviation dictates how much the signal deviates from the reference on average. It can be observed that when disturbances are influenced the system the system is less affected if the super twisting SMC algorithm is applied. This fact may conclude that more accuracy is achieved when tracking trajectories in machining and better accuracy in the final product may be achieved.

\section{CONCLUSIONS}

In this paper, two different schemes have been analysed in order to deal with the motion tracking of a linear motor actuated in micro-milling processes. The micro-milling cutting forces act as disturbances into the linear motor which is composed by mass and damper against inertia of the system. The following key findings can be pointed out:

- Super twisting SMC control scheme is more stable and robust against variations in plant model parameters, influence of external perturbations and tuneable parameters of the control itself than the P-PI position and velocity control scheme as the simulation results show.

- The errors in position and velocity are less influenced under disturbances when the super-twisting SMC is applied to the linear motor system. This may lead to better tracking the reference signal and better accuracy in the final product of the system.

- The proposed controllers are able to achieve submicron accuracy single axis motion tracking depending upon the resolution positioning sensor and tuning of the controller parameters. More resolution the sensor has, better positioning can be achieved.

\section{ACKNOWLEDGMENT}

The authors gratefully acknowledge the financial support from the EPSRC (EP/K018345/1) for this study. A. Ibeas is very grateful to the Spanish Government for its partial support to this work through grant DPI2013-47825-C3-1-R.

\section{REFERENCES}

[1] Y., Altintas, K., Erkorkmaz and W-H., Zhu (2000), Sliding mode controller design for high speed feed drives, Annals of the CIRP 49 (1): 265-270.

[2] V. I., Utkin (1992), Sliding modes in control and optimization, NY, Berlin: Springer-Verlag.

[3] V. I., Utkin and A. S. Poznyak (2013), Adaptive sliding mode control with application to super-twist algorithm: Equivalent control method, Automatica, Vol. 49, 39-47.

[4] Y., Altintas and C.E., Okwudire (2009), Dynamic stiffness enhancement of direct-driven machine tools using sliding mode control with disturbance recovery, CIRP Annals, Manufacturing Technology 58:335338.

[5] F. J., Villegas, R. L., Hecker, M. E., Pena, D. A., Vicente and G. M., Flores, (2014), Modelling a linear motor feed drive including pre-rolling friction and aperiodic cogging and ripple, International J Adv. Manuf. Technology, 73, 267-277. 
[6] Y., Altintas (2013), Manufacturing Automation: Metal cutting mechanics, machine tool vibrations, and CNC design, $2^{\text {nd }}$ ed., Cambridge University Press.

[7] B., Wu (2013), Analysis and simulation of micro-milling process, Master Engineering Thesis, Faculty of engineering, University of South Wales.

[8] I., Eker (2006), Sliding mode control with PID sliding surface and experimental application to an electromechanical plant, ISA transactions, vol. 45, pp. 109-118.
[9] X., Yu and M., Önder Efe (Ed.) (2015), Recent advances in sliding modes: from control to intelligent mechatronics, Studies in systems, decision and control, 24, Springer.

[10] Y., Li and Q., Xu, (2010), Adaptive sliding mode control with perturbation estimation and PID sliding surface for motion tracking of a piezo-driven micromanipulator, IEEE Transactions on control systems technology, 18 (4)798-810.

[11] X-C., Xi, W-S., Zhao, and A-N., Poo, (2015), Improving CNC contouring accuracy by robust digital integral sliding mode control, International Journal of Machine Tools \& Manufacturing 88:51-61. 